\title{
MEDIDAS DE PREVENÇÃO DA PNEUMONIA ASSOCIADA À VENTILAÇÃO MECÂNICA: UMA ANÁLISE À LUZ DA LITERATURA CIENTÍFICA
}

\section{PREVENTION MEASURES FOR PNEUMONIA ASSOCIATED WITH MECHANICAL VENTILATION: AN ANALYSIS IN THE LIGHT OF SCIENTIFIC LITERATURE}

\author{
Joaquim Ismael de Sousa Teixeira ${ }^{1} *$ Raimunda Leandra Bráz da Silva $^{2}$
}

\section{RESUMO}

Objetivo: O objetivo deste estudo foi analisar as medidas de prevenção de Pneumonia associada à Ventilação Mecânica em Unidades de Terapia Intensiva. Método: Trata-se de uma revisão integrativa realizada em janeiro de 2021. A busca dos artigos ocorreu a partir das bases de dados: Scientific Electronic Library Online (SciELO); Literatura Latino-Americana e do Caribe em Ciências da Saúde (LILACS) e Base de Dados em Enfermagem (BDENF). Foram incluídos artigos de pesquisa original, disponíveis na íntegra, em idioma português, inglês e espanhol, com publicação nos últimos 03 anos. Foram excluídos estudos que não retratavam a realidade de UTI adulto ou não respondessem à questão norteadora. Ao final, 11 artigos foram selecionados. Resultados e Discussão :Em relação às medidas de prevenção de PAV citadas: decúbito elevado em $30^{\circ}$ a $45^{\circ}$ esteve presente em todos os estudos analisados (100\%). Higiene oral com Clorexidina $0,12 \%(90,9 \%)$ e Pressão do cuff entre 20 e $30 \mathrm{~mm} \mathrm{H} 20$ (81,8\%) foram outras medidas que se destacaram. Conclusão: Percebe-se que as medidas de prevenção da PAV são bem disseminadas e apresentam um consenso no âmbito científico, o desafio, porém está no acompanhamento da adesão dos profissionais a esse conjunto de ações, o que reverbera diretamente nas taxas de infecção e segurança do paciente.

Palavras-chave: Infecção Hospitalar; Pneumonia associada à Ventilação Mecânica; Unidades de Terapia Intensiva.

\begin{abstract}
Objective: The objective of this study was to analyze the preventive measures for Pneumonia associated with Mechanical Ventilation in Intensive Care Units. Method: This is an integrative review conducted in January 2021. The search for the articles occurred from the databases: Scientific Electronic Library Online (SciELO); Latin American and Caribbean Literature on Health Sciences (LILACS) and Nursing Database (BDENF). Original research articles were included, available in full, in Portuguese, English and Spanish, with publication in the last 03 years. Studies that did not portray the reality of adult ICU or did not answer the guiding question were excluded. At the end, 11 articles were selected. Results and Discussion: Regarding the aforementioned VAP prevention measures: decubitus elevated from $30^{\circ}$ to $45^{\circ}$ was present in all studies analyzed (100\%). Oral hygiene with Chlorhexidine 0.12\% (90.9\%) and Cuff pressure between 20 and $30 \mathrm{~mm} \mathrm{H} 20$ $(81.8 \%)$ were other measures that stood out. Conclusion: It is noticed that the measures to prevent VAP are well disseminated and have a consensus in the scientific field, the challenge, however, is in monitoring the adherence of professionals to this set of actions, which directly reverberates in the rates of infection and safety of the patient.

Keywords: Hospital Infection; Pneumonia associated with Mechanical Ventilation; Intensive Care Units

\footnotetext{
${ }^{1}$ Enfermeiro pela Universidade Estadual Vale do Acaraú (UVA), Sobral, Ceará, Brasil. Mestrando do Programa de Pós Graduação em Saúde da Família da Universidade Federal do Ceará (UFC), campus Sobral, Ceará. Email: joaquimismaeldesousateixeira@gmail.com ID ORCID: https://orcid.org/0000-0002-5027-8030

${ }^{2}$ Enfermeira pela Universidade Estadual Vale do Acaraú (UVA), Sobral, Ceará, Brasil. Residente em Urgência e Emergência pela Santa Casa de Misericórdia de Sobral (SCMS). E-mail: leandrabraz7@gmail.com ID ORCID: https://orcid.org/0000-0002-0819$\underline{5987}$
} 


\section{INTRODUÇÃO}

Segundo a Agência Nacional de Vigilância Sanitária (ANVISA), as Infecções Hospitalares (IH), são definidas como toda causa infecciosa contraída pelo paciente após sua admissão em um ambiente hospitalar ${ }^{(1)}$. Em termos de incidência, estima-se que, a cada 100 pacientes hospitalizados, 10 serão acometidos por IH, ocasionando problemas éticos, jurídicos e sociais, além de prolongamento do tempo de internação e em casos mais graves acarretando óbitos ${ }^{(2)}$.

Entre os diversos agravos que afetam a qualidade do cuidado e impactam negativamente na segurança do paciente destacam-se as Infecções Relacionadas à Assistência à Saúde (IRAS). As IRAS aumentam o tempo de internação, elevam os custos assistenciais e até mesmo, a morbimortalidade $^{(3)}$. Essas infecções podem se manifestar durante a internação ou após a alta hospitalar ${ }^{(4)}$.

A utilização de procedimentos cada vez mais aprimorados, a patogenicidade dos microrganismos e o uso inadvertido de antimicrobianos resultando no aparecimento da resistência microbiana, são os principais responsáveis pelo aumento nos índices de IRAS. Entre essas infecções destaca-se a Pneumonia Associada a Ventilação Mecânica (PAV), com resultados negativos tanto para o paciente internado na Unidade de Terapia Intensiva (UTI) como para as instituições de saúde, aumentando a morbidade e a mortalidade entre os pacientes ${ }^{(5)}$.

A PAV é a infecção que ocorre 48 horas após a intubação e/ou 72 horas após a extubação, cujos agentes etiológicos não estavam presentes no período da admissão do paciente. Surge como uma infecção que acomete pacientes críticos ventilados mecanicamente na UTI. Dados epidemiológicos evidenciam que a PAV apresenta incidência entre 10 e $30 \%$ do total de pacientes internados na UTI. Além disso, está associada a hospitalização prolongada, aumento dos custos com cuidados de saúde e mortalidade variando entre $8,1 \%$ a $31,9 \%$, representando um desafio terapêutico ${ }^{(6)}$.

Essas infecções são consideradas mais graves na UTI, em que são atendidos pacientes dependentes de suporte intensivo de vida. Neste ambiente, o paciente está mais exposto ao risco de infecção, haja vista sua condição clínica e a variedade de procedimentos invasivos rotineiramente realizados. Em UTI, os pacientes têm de 5 a 10 vezes mais probabilidades de contrair infecção e esta pode representar cerca de $20 \%$ do total das infecções de um hospital ${ }^{(7)}$.

Consonante a isso, o Ministério da Saúde (MS) implantou por meio da Portaria número 2.216 de 1998, a Comissão de Controle de Infecções Hospitalares (CCIH), que trabalha na redução da incidência e gravidade na evolução dos pacientes internados, redução do custo hospitalar por meio do controle e prevenção de IRAS ${ }^{(8)}$.

A CCIH tem um papel importante nas instituições hospitalares na busca de prevenção e controle das infecções, em que desenvolve um conjunto de ações deliberadas e sistemáticas, com vistas à redução máxima da incidência e gravidade destas, sendo o profissional enfermeiro, o profissional ideal para compor a equipe, com habilidades de gerenciamento, avaliação da qualidade dos serviços e práticas assistenciais ${ }^{(9)}$.

Face à importância e à complexidade da PAV, é essencial a realização de medidas que causem impacto na prevenção, levando à redução da ocorrência de infecção. A utilização destas recomendações tem como relevância a variabilidade de práticas, em que as suas intervenções estejam baseadas em evidências científicas, acarretando a redução 
das taxas de incidência, aprimorando os cuidados prestados e, por conseguinte, aportando um melhor prognóstico do doente crítico.

Diante do exposto, é fundamental que ações de prevenção sejam prioritárias nas instituições de saúde, a fim de, promover segurança aos pacientes que necessitam de assistência ventilatória invasiva durante sua internação em UTI. Além de permitir a identificação dos cuidados de prevenção da PAV, torna-se relevante observar a percepção dos envolvidos quanto a essa problemática e o mapeamento das condições atuais que poderão ser trabalhadas em espaços educativos, dessa forma, contribuindo para o fortalecimento de ações de promoção e prevenção.

Este estudo tem como objetivo analisar as medidas de prevenção e controle de Pneumonia associada à Ventilação Mecânica realizadas em Unidades de Terapia Intensiva descritas na literatura internacional.

\section{MÉTODO}

A presente pesquisa trata-se de uma revisão integrativa de literatura, com abordagem quanti-qualitativa, realizada no período de janeiro de 2021. Esse tipo de estudo busca sintetizar os resultados de pesquisas internacionalmente reconhecidas na área temática abordada. Além disso, é uma ferramenta importante na saúde por fomentar a utilização de evidências científicas presentes com repercussões para a prática profissional $^{(10)}$.

A realização da busca dos artigos para comporem a revisão ocorreu por meio de revisão por pares, a partir das seguintes bases de dados: Scientific Electronic Library Online (SciELO); Literatura Latino-Americana e do
Caribe em Ciências da Saúde (LILACS) e Base de Dados em Enfermagem (BDENF).

A escolha pelas bases citadas deu-se pelo amplo escopo de estudos internacionalmente conhecidos, a fim de se conseguir as melhores e mais atuais evidências a respeito do tema. Para as buscas, fez-se o uso: das palavras-chave "Pneumonia Associada à Ventilação Mecânica" e "Prevenção"; e do descritor via Descritores em Ciências da Saúde (DeCS), "Unidades de Terapia Intensiva". O cruzamento de ambas foi realizado por meio do operador boleano "AND".

As buscas ocorreram de forma a serem mais fidedignas e não fugisse ao tema principal pesquisado, o que foi norteado pela seguinte questão norteadora: Quais as medidas de prevenção e controle de Pneumonia Associada à Ventilação Mecânica são mais utilizadas atualmente em ambientes de Terapia Intensiva?

Como critérios de inclusão utilizaramse da filtragem por artigos científicos, de pesquisa original, disponíveis na íntegra, em idioma português, inglês e espanhol, com publicação nos últimos 03 anos (2018-2020). Foram excluídos aqueles estudos que não retratavam a realidade de Unidades de Terapia Intensiva com internação do público adulto ou que não respondessem a pergunta de pesquisa a respeito das medidas de prevenção e controle da PAV nesses ambientes.

No cruzamento "Pneumonia associada à Ventilação Mecânica" and "Unidades de Terapia Intensiva" realizado sem filtros, encontrou-se os seguintes resultados: 34 estudos na SciELO, 29 na BDENF e 101 na LILACS, totalizando 164 resultados. Após aplicação dos filtros, 130 estudos foram excluídos da amostra, bem como 29 encontravam-se duplicados entre as 3 bases de dados. Para análise completa restaram 5 
artigos, destes 1 não se adequava a temática do estudo após leitura do título e resumo, Já em relação a "Prevenção" and "Pneumonia associada à Ventilação Mecânica", 135 resultados foram encontrados, sendo 25 na SciELO, 35 na BDENF e 75 na LILACS. Após a filtragem, 105 estudos foram excluídos e 19 estavam duplicados nas buscas. Para análise completa dos 11 artigos restantes, 2 estudos foram excluídos por serem artigos de revisão e não estudos originais, bem como 1 artigo não retratava o restando assim 4 estudos para comporem a amostra.

ambiente de UTI e 1 artigo trazia o contexto de UTI pediátrica e não de adultos. Dessa forma, 7 estudos foram escolhidos para comporem a amostra. Ao final, com a união dos resultados dos dois cruzamentos, 11 artigos foram selecionados para comporem a revisão. A figura 01 apresenta segundo a metodologia PRISMA os artigos que compuseram a revisão integrativa.

Figura 1 - Prisma de buscas Scielo, LILACS e BDENF. Sobral, Ceará, Brasil, 2021.

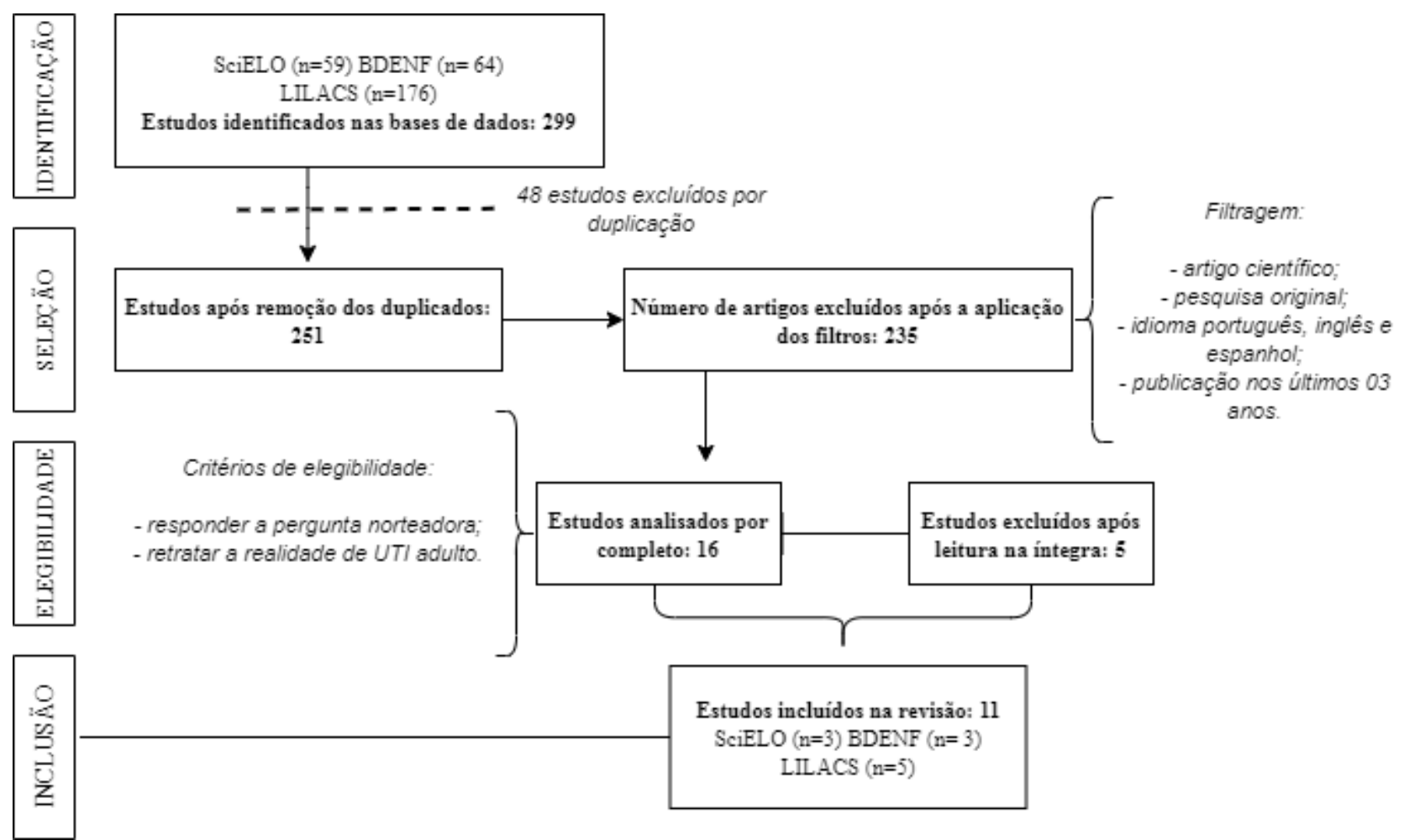

Fonte: Elaboração própria.

\section{RESULTADOS E DISCUSSÃO}

A partir dos estudos selecionados, foi realizada a análise dos mesmos a partir do instrumento de Ursi ${ }^{(11)}$ adaptado pelos autores conforme o quadro 1 a seguir. Utilizou-se como parâmetros: título, autor/ ano, idioma, objetivo do estudo, área de publicação, tipo de estudo e medidas de prevenção e controle da PAV citadas nos estudos. 
QUADRO 1. Instrumento para análise dos estudos. Sobral, Ceará, Brasil, 2021.

\begin{tabular}{|c|c|c|c|c|c|c|c|}
\hline $\mathbf{N}^{\circ}$ & Título & Autor/ Ano & Idioma & Objetivo & $\begin{array}{c}\text { Área de } \\
\text { Publicação }\end{array}$ & Tipo de Estudo & $\begin{array}{l}\text { Medidas de Prevenção e } \\
\text { Controle da PAV }\end{array}$ \\
\hline I. & $\begin{array}{c}\text { Boas práticas na } \\
\text { prevenção de } \\
\text { pneumonia associada à } \\
\text { ventilação mecânica }\end{array}$ & $\begin{array}{l}\text { Alecrim et } \\
\text { al., } 2019\end{array}$ & Português & $\begin{array}{l}\text { Avaliar a adesão dos profissionais } \\
\text { de saúde a um conjunto de boas } \\
\text { práticas de prevenção de } \\
\text { Pneumonia Associada à } \\
\text { Ventilação } \\
\text { Mecânica, índice de } \\
\text { conformidade às medidas } \\
\text { individuais e associação de } \\
\text { características clínicas dos } \\
\text { pacientes e adesão ao conjunto de } \\
\text { boas práticas com a pneumonia. }\end{array}$ & Enfermagem & $\begin{array}{l}\text { Estudo de coorte } \\
\text { prospectivo }\end{array}$ & $\begin{array}{c}\text { - Avaliação diária da } \\
\text { sedação e redução sempre } \\
\text { que possível; } \\
\text { - Troca do circuito a cada } 7 \\
\text { dias; } \\
\text { - Manutenção da pressão do } \\
\text { cuff entre } 20 \text { e 30mm H2O; } \\
\text { - Decúbito elevado (30 } \\
\text { a 45º); } \\
\text { - Higiene oral com } \\
\text { Clorexidina solução } \\
\text { 0,12\% (3 vezes ao dia). }\end{array}$ \\
\hline II. & $\begin{array}{l}\text { Adesão ao bundle de } \\
\text { prevenção de } \\
\text { pneumonia associada à } \\
\text { ventilação mecânica. }\end{array}$ & $\begin{array}{c}\text { Barros, } \\
2019\end{array}$ & Português & $\begin{array}{l}\text { Avaliar a adesão e conformidade } \\
\text { das práticas que integram um } \\
\text { bundle de prevenção da } \\
\text { Pneumonia } \\
\text { Associada à Ventilação Mecânica } \\
\text { em uma Unidade de Terapia } \\
\text { Intensiva de adulto. }\end{array}$ & Enfermagem & $\begin{array}{c}\text { Estudo descritivo } \\
\text { com abordagem } \\
\text { prospectiva }\end{array}$ & $\begin{array}{c}\text { - Posicionamento da } \\
\text { cabeceira entre } 30 \text { e } 45^{\circ} \text {; } \\
\text { - Pressão do cuff entre } 20-30 \\
\text { cm H2O; } \\
\text { - Higiene oral com } \\
\text { clorexidina } 0,12 \% ;\end{array}$ \\
\hline
\end{tabular}

https://doi.org/10.31011/reaid-2021-v.95-n.34-art.1018 Rev Enferm Atual In Derme v. 95, n. 34, 2021 e-021056 


\begin{tabular}{|c|c|c|c|c|c|c|c|}
\hline & & & & & & & $\begin{array}{l}\text { - Fisioterapia respiratória e } \\
\text { motora; } \\
\text { - Cuidados com a aspiração } \\
\text { das secreções; } \\
\text { - Interrupção da infusão de } \\
\text { sedativos. }\end{array}$ \\
\hline III. & $\begin{array}{l}\text { Education to prevent } \\
\text { Ventilator associated } \\
\text { pneumonia in Intensive } \\
\text { Care Unit. }\end{array}$ & $\begin{array}{l}\text { Branco et } \\
\text { al., } 2020\end{array}$ & Português & $\begin{array}{l}\text { Avaliar a adesão da enfermagem } \\
\text { ao bundle de prevenção à } \\
\text { Pneumonia Associada à } \\
\text { Ventilação Mecânica e a taxa de } \\
\text { incidência, antes e após Educação } \\
\text { Permanente. }\end{array}$ & Enfermagem & $\begin{array}{l}\text { Estudo quase } \\
\text { experimental, } \\
\text { retrospectivo. }\end{array}$ & $\begin{array}{l}\text { - Posição do filtro do } \\
\text { ventilador mecânica acima } \\
\text { da inserção do tubo } \\
\text { endotraqueal ou } \\
\text { traqueostomia, ausente de } \\
\text { sujidades; } \\
\text { - Cabeceira do leito elevada } \\
\text { entre } 30^{\circ} \text { e } 45^{\circ} \text {; } \\
\text { - Realização da higiene oral } \\
\text { com clorexidina } 0,12 \% \text { e } \\
\text { escovação dos dentes; - } \\
\text { Pressão do balonete, entre } \\
20 \text { e } 30 \text { mmHg. }\end{array}$ \\
\hline IV. & $\begin{array}{c}\text { O impacto da } \\
\text { aprendizagem } \\
\text { interprofissional na } \\
\text { pneumonia associada à } \\
\text { ventilação: } \\
\text { implementação de } \\
\text { bundles em uma } \\
\text { unidade de cuidados }\end{array}$ & $\begin{array}{l}\text { Coelho et } \\
\text { al., } 2019\end{array}$ & Português & $\begin{array}{c}\text { Verificar o impacto dos bundles e } \\
\text { o aprendizado interprofissional na } \\
\text { prevenção de pneumonia } \\
\text { associada a ventilação mecânica } \\
\text { de uma unidade de terapia } \\
\text { intensiva. }\end{array}$ & Enfermagem & $\begin{array}{l}\text { Estudo quase- } \\
\text { experimental. }\end{array}$ & $\begin{array}{c}\text { - Higiene das mãos; } \\
\text { - Elevação do leito a 30-45; } \\
\text { - Interrupções diárias da } \\
\text { sedação; } \\
\text { - Testes diários de respiração }\end{array}$ \\
\hline
\end{tabular}




\begin{tabular}{|c|c|c|c|c|c|c|c|}
\hline & intensivos & & & & & & $\begin{array}{c}\text { espontânea; } \\
\text { - Profilaxia do } \\
\text { tromboembolismo; } \\
\text { - Higiene bucal com } \\
\text { gluconato de clorexidina; } \\
\text { - Drenagem de secreção } \\
\text { subglotica; } \\
\text { - Monitoramento constante } \\
\text { da pressão do cuff; } \\
\text { - Educação Permanente. }\end{array}$ \\
\hline V. & $\begin{array}{l}\text { Pneumonia associada à } \\
\text { ventilação mecânica } \\
\text { invasiva: cuidados de } \\
\text { enfermagem }\end{array}$ & $\begin{array}{l}\text { Cruz; } \\
\text { Martins, } \\
2019\end{array}$ & Português & $\begin{array}{l}\text { Identificar os procedimentos de } \\
\text { enfermagem em doentes } \\
\text { submetidos à ventilação mecânica } \\
\text { invasiva e o desenvolvimento de } \\
\text { pneumonia num serviço de } \\
\text { medicina intensiva. }\end{array}$ & Enfermagem & $\begin{array}{c}\text { Estudo } \\
\text { longitudinal e } \\
\text { descritivo }\end{array}$ & $\begin{array}{c}\text { - Bundles; } \\
\text { - Elevação da cabeceira da } \\
\text { cama; } \\
\text { - Higienização das mãos; } \\
\text { - Aspiração de secreções; } \\
\text { - Higiene oral; } \\
\text { - Manutenção dos circuitos } \\
\text { ventilatórios; } \\
\text { - Avaliação da pressão do } \\
\text { cuff. }\end{array}$ \\
\hline VI. & & Dutra et al., & Inglês & $\begin{array}{l}\text { Apreender a percepção dos } \\
\text { profissionais de Enfermagem }\end{array}$ & Enfermagem & $\begin{array}{l}\text { Estudo } \\
\text { qualitativo, }\end{array}$ & - Higienização das mãos; \\
\hline
\end{tabular}

https://doi.org/10.31011/reaid-2021-v.95-n.34-art.1018 Rev Enferm Atual In Derme v. 95, n. 34, 2021 e-021056 


\begin{tabular}{|c|c|c|c|c|c|c|c|}
\hline & $\begin{array}{l}\text { Ventilator-Associated } \\
\text { Pneumonia: perception } \\
\text { of the nursing staff }\end{array}$ & 2019 & & $\begin{array}{l}\text { sobre a segurança do paciente sob } \\
\text { ventilação mecânica com vistas à } \\
\text { prevenção da PAV. }\end{array}$ & & $\begin{array}{l}\text { descritivo e } \\
\text { exploratório }\end{array}$ & $\begin{array}{l}\text { - Manutenção de decúbito } \\
\text { elevado entre } 30 \text { e } 45^{\circ} ; \\
\text { - Adequação diária do nível } \\
\text { de sedação e do teste de } \\
\text { respiração espontânea; } \\
\text { - Aspiração subglótica; } \\
\text { - Higiene oral com } \\
\text { antissépticos; } \\
\text { - Cuidados com o circuito do } \\
\text { ventilador, inaladores e } \\
\text { nebulizadores; } \\
\text { - Monitorização da pressão } \\
\text { de cuff. }\end{array}$ \\
\hline VII. & $\begin{array}{l}\text { Good practices for } \\
\text { preventing ventilator- } \\
\text { associated pneumonia } \\
\text { in the emergency } \\
\text { department }\end{array}$ & $\begin{array}{l}\text { Frota et al., } \\
2019\end{array}$ & Inglês & $\begin{array}{l}\text { Avaliar a conformidade do } \\
\text { conjunto de boas práticas para } \\
\text { prevenção da pneumonia } \\
\text { associada à ventilação mecânica } \\
\text { no serviço de urgência e } \\
\text { emergência de um hospital } \\
\text { universitário. }\end{array}$ & Enfermagem & $\begin{array}{c}\text { Estudo } \\
\text { transversal, } \\
\text { analítico e de } \\
\text { abordagem } \\
\text { quantitativa. }\end{array}$ & $\begin{array}{l}\text { - Elevação da cabeceira de } \\
\qquad 30^{\circ} \text { a } 45^{\circ} ; \\
\text { - Interrupção diária da } \\
\text { sedação; } \\
\text { - Profilaxia de úlcera péptica } \\
\text { e trombose venosa profunda } \\
\text { (TVP); } \\
\text { - Higiene oral com } \\
\text { clorexidina; } \\
\text { - Aspiração de secreção } \\
\text { subglótica; } \\
\text { - Mensuração da pressão do }\end{array}$ \\
\hline
\end{tabular}

https://doi.org/10.31011/reaid-2021-v.95-n.34-art.1018 Rev Enferm Atual In Derme v. 95, n. 34, 2021 e-021056 


\begin{tabular}{|c|c|c|c|c|c|c|c|}
\hline & & & & & & & cuff. \\
\hline VIII. & $\begin{array}{l}\text { Cuidados } \\
\text { multiprofissionais } \\
\text { relacionados à } \\
\text { prevenção da } \\
\text { pneumonia associada à } \\
\text { ventilação mecânica }\end{array}$ & $\begin{array}{l}\text { Liz et al., } \\
2020\end{array}$ & Português & $\begin{array}{l}\text { Conhecer a percepção da equipe } \\
\text { multiprofissional acerca dos } \\
\text { cuidados relacionados às medidas } \\
\text { de prevenção a pneumonia } \\
\text { associada a ventilação mecânica. }\end{array}$ & Enfermagem & $\begin{array}{c}\text { Estudo descritivo, } \\
\text { qualitativo. }\end{array}$ & $\begin{array}{c}\text { - Higiene Oral; } \\
\text { - Mudança de decúbito; } \\
\text { - Atuação próxima da CCIH; } \\
\text { - Avaliação da vias aéreas; } \\
\text { - Educação Permanente. }\end{array}$ \\
\hline IX. & $\begin{array}{l}\text { Ventilator-Associated } \\
\text { Pneumonia: the } \\
\text { Knowledge of Health } \\
\text { Professionals Towards } \\
\text { Prevention and } \\
\text { Educational Measures }\end{array}$ & $\begin{array}{l}\text { Melo et al., } \\
2019\end{array}$ & Inglês & $\begin{array}{c}\text { Avaliar o conhecimento dos } \\
\text { profissionais de saúde sobre a } \\
\text { prevenção da Pneumonia } \\
\text { Associada à Ventilação Mecânica } \\
\text { em pacientes críticos internados } \\
\text { nas Unidades de Terapia Intensiva } \\
\text { e, promover educação permanente } \\
\text { para profissionais das UTIs sobre } \\
\text { prevenção de PAVM. }\end{array}$ & Enfermagem & $\begin{array}{c}\text { Estudo } \\
\text { transversal, } \\
\text { quanti-qualitativo. }\end{array}$ & $\begin{array}{l}\text { - Higienização das mãos; } \\
\text { - Elevação da cabeceira; } \\
\text { - Pressão do cuff; } \\
\text { - Fisioterapia respiratória; } \\
\text { - Higienização oral; } \\
\text { - Retirada de sedação; } \\
\text { - Educação Permanente. }\end{array}$ \\
\hline $\mathbf{X}$. & $\begin{array}{l}\text { Adesão às medidas } \\
\text { preventivas versus } \\
\text { incidência de } \\
\text { pneumonia associada à } \\
\text { ventilação mecânica }\end{array}$ & $\begin{array}{c}\text { Silva } \\
\text { Lourençone } \\
\text { et al., } 2019\end{array}$ & Português & $\begin{array}{l}\text { Avaliar a taxa de adesão das } \\
\text { ações preventivas da equipe de } \\
\text { enfermagem para PAV, após a } \\
\text { reestruturação e aplicação do } \\
\text { protocolo de prevenção e verificar } \\
\text { as taxas de densidade de } \\
\text { incidência de pacientes com PAV. }\end{array}$ & Enfermagem & $\begin{array}{c}\text { Estudo } \\
\text { observacional, } \\
\text { longitudinal. }\end{array}$ & $\begin{array}{c}\text { - Bundle; } \\
\text { - Higiene oral com } \\
\text { clorexidina } 0,12 \% \text { duas } \\
\text { vezes ao dia, às } 8 \text { h e às } 20 \\
\text { horas; } \\
\text { - Cabeceira elevada em } 30 \text { a } \\
45^{\circ} ; \\
\text { - Pressão do balonete }\end{array}$ \\
\hline
\end{tabular}

https://doi.org/10.31011/reaid-2021-v.95-n.34-art.1018 Rev Enferm Atual In Derme v. 95, n. 34, 2021 e-021056 


\begin{tabular}{|c|c|c|c|c|c|c|c|}
\hline & & & & & & & $\begin{array}{c}\text { avaliada duas vezes ao dia, } \\
\text { no mesmo horário da higiene } \\
\text { oral; } \\
\text { - Posição do filtro do } \\
\text { circuito do ventilador } \\
\text { posicionada acima de sua } \\
\text { inserção do tubo e sem } \\
\text { presença de sujidade. }\end{array}$ \\
\hline XI. & $\begin{array}{l}\text { Adesão ao protocolo de } \\
\text { pneumonia associado à } \\
\text { ventilação mecânica }\end{array}$ & $\begin{array}{c}\text { Zigart et al., } \\
2019\end{array}$ & Inglês & $\begin{array}{l}\text { Conhecer a adesão da equipe de } \\
\text { enfermagem ao protocolo de } \\
\text { pneumonia associada à ventilação } \\
\text { mecânica nas Unidades de } \\
\text { Terapia Intensiva. }\end{array}$ & Enfermagem & $\begin{array}{c}\text { Estudo } \\
\text { quantitativo, } \\
\text { descritivo, } \\
\text { observacional, } \\
\text { transversal. }\end{array}$ & $\begin{array}{l}\text { - Manter o decúbito elevado } \\
\text { entre } 30 \text { a } 45^{\circ} ; \\
\text { - Filtro trocador de calor e } \\
\text { umidade (Heat and Moisture } \\
\text { Exchangers - HME); } \\
\text { - Cuidados com o sistema de } \\
\text { aspiração, inalação e } \\
\text { nebulização. }\end{array}$ \\
\hline
\end{tabular}


Dos 11 artigos analisados, 2 destes $(18,2 \%)$ tem data de publicação de 2020 e 9 são do ano 2019 (81,8\%). Não foram evidenciados estudos do ano de 2018 após as buscas realizadas.

Em relação aos idiomas dos estudos analisados, $63,6 \%$ destes $(n=7)$ estavam disponíveis em português na versão original, e $36,4 \%(n=4)$ em idioma inglês. Não foram encontrados estudos em língua espanhola, embora alguns estivessem disponíveis no idioma em versões traduzidas.

Sobre a área de publicação, $100 \%$ dos artigos foram de Enfermagem. Embora a busca tenha ocorrido em uma base de dados voltada a esse público, BDENF, percebe-se a mesma tendência de publicação na área nas demais bases, confirmando assim o que traz o $\operatorname{artigo~}^{(12)}$ sobre a importância da enfermagem na gerência e coordenação das medidas de prevenção da PAV numa lógica multiprofissional alicerçada em evidências científicas.

Dos tipos de estudo presentes na amostra, 72,7\% $(\mathrm{n}=8)$ se enquadram como do tipo descritivos/ transversais. Estudos quase experimentais $(18,2 \%)$ apareceram duas vezes e um dos artigos analisados se tratava de um estudo de coorte $(9,1 \%)$.

A Figura 2 a seguir demonstra 0 gráfico da incidência das medidas de prevenção de PAV que mais se repetiram dentre os estudos analisados.

Figura 2 - Incidência de citação das medidas de prevenção de PAV. Sobral, Ceará, Brasil, 2021.

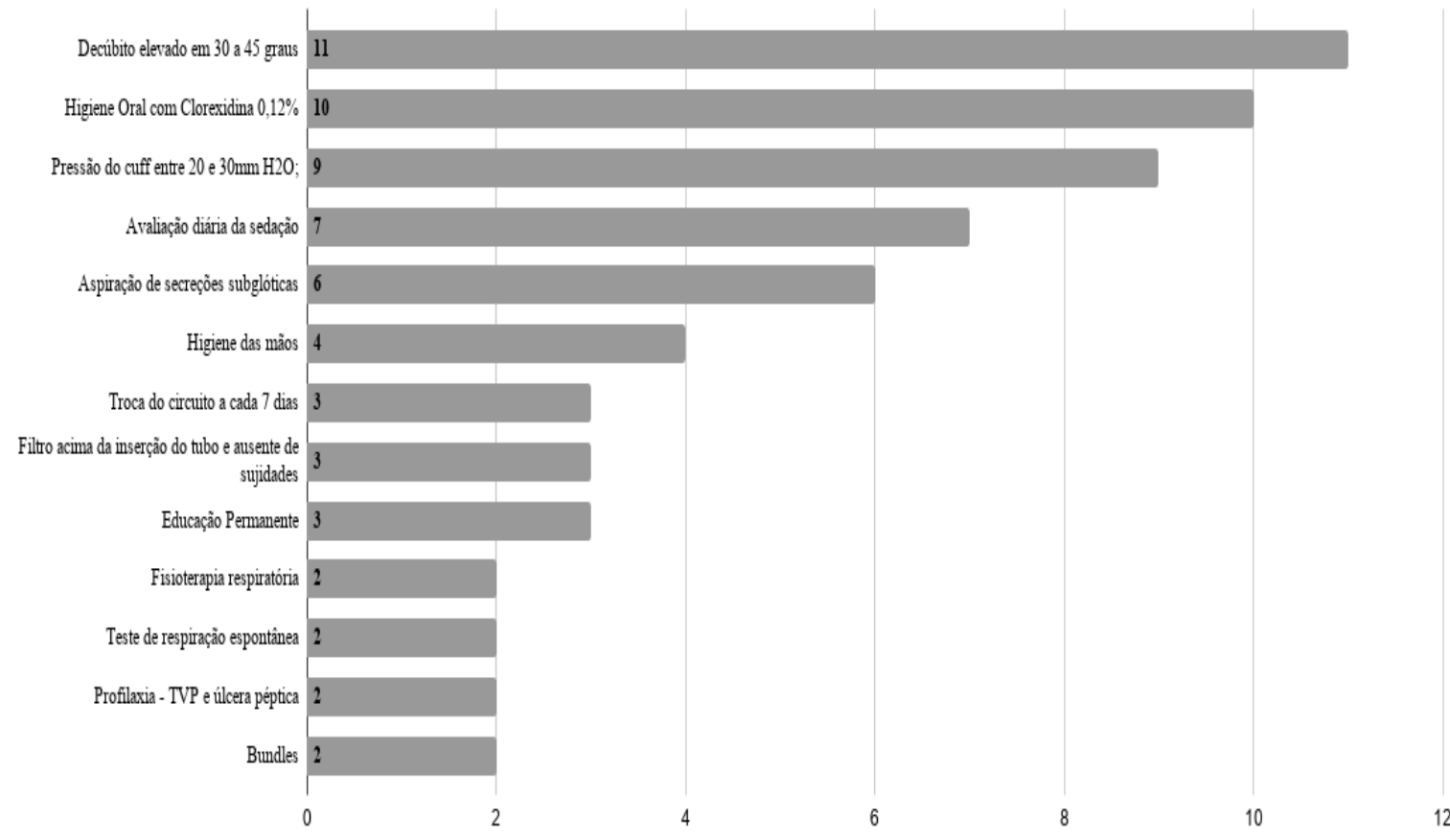

Fonte: Elaboração própria. 
Em relação à incidência das medidas de prevenção de PAV citadas nos artigos da revisão, Decúbito elevado em 30 a 45 graus foi a que mais se repetiu entre os artigos, estando presentes em todos os estudos analisados (100\%). Higiene oral com Clorexidina $0,12 \%$ em 10 dos 11 artigos (90,9\%) e Pressão do cuff entre 20 e $30 \mathrm{~mm}$ H20 em 9 de 11 artigos $(81,8 \%)$ foram outras medidas que mais se destacaram entre aquelas preconizadas para prevenção desse tipo de infecção.

Para a discussão dos estudos, foi realizada a análise temática dos mesmos e junto à literatura científica atual disponível foi discorrido a respeito das evidências científicas sobre medidas de prevenção e controle da PAV.

\section{Boas práticas de prevenção da Pneumonia Associada à Ventilação Mecânica em Cuidados Intensivos: o que há de mais atual?}

O uso prolongado de ventilação mecânica predispõe os pacientes a desenvolver eventos adversos como sepse, síndrome da angustia respiratória aguda (SARA), embolia pulmonar, barotrauma, edema pulmonar e o principal deles, a Pneumonia Associada à Ventilação Mecânica $^{(3)}$.

Os autores ${ }^{(13)}$ classificam os fatores de risco para o desenvolvimento da PAV como modificáveis e não modificáveis: estes últimos estão relacionados à idade e às condições de saúde do paciente. Já os primeiros, incluem: tempo prolongado de ventilação mecânica, intubações endotraqueais subsequentes, sondagem nasogástrica, imobilização, posicionamento supino, uso de agentes antimicrobianos, uso de medicamentos antiácidos, sedação, inadequada higienização das mãos pelos profissionais e os maus cuidados de assepsia durante a intubação, a aspiração de vias aéreas e o manuseio do respirador.

São exatamente esses fatores modificáveis, que a aplicação de um pacote de medidas pode reduzir significativamente as taxas de PAV, principalmente nos setores de cuidados intensivos ao prevenir esse tipo de infecção. O conjunto dessas ações pode estar de forma mais simplificada e sucinta, no formato comumente chamado de bundles, a fim de facilitar o entendimento e a adesão dos profissionais a esses procedimentos. $\mathrm{O}$ acompanhamento desses indicadores de adesão é indispensável para o controle das taxas de densidade e incidência da PAV ${ }^{(14)}$.

Porém, os bundles, isoladamente, não asseguram uma redução nas taxas de PAV, devendo, portanto, serem implementadas ações educativas de treinamento e capacitação da equipe, bem como ofertar feedback do acompanhamento das taxas de adesão e incidência da PAV a fim da reflexão crítica da equipe em relação as suas práticas diárias ${ }^{(7)}$.

Nesse processo, a Enfermagem assume papel central, como profissão que atua diretamente no cuidado ao paciente crítico, além de ser a equipe de enfermagem a responsável pela maior parte das ações propostas como preventivas ao desenvolvimento da $\mathrm{PAV}^{(15)}$. Por isso, corroborando ao que os autores $^{(6,16)}$ trazem nos artigos III e VIII, é indispensável o envolvimento do enfermeiro, como líder dessa equipe, nos processos formativos em relação à prevenção das IRAS, além do contato direto junto ao responsável pela $\mathrm{CCIH}$ do hospital na adoção dessas medidas. 
Para quantificar a importância da estratégia de bundles e o acompanhamento das taxas de adesão ao check-list, dos 11 artigos selecionados desta revisão, a palavra "bundle" está presente nos objetivos de 3 destes $^{(16-18)}$, e em outros 4 artigos, os objetivos buscam avaliar a adesão dos profissionais ao conjunto de boas práticas de prevenção da $\mathrm{PAV}^{(19-22)}$. Totalizando assim um percentual de $63,6 \%$ de estudos na amostra que tratam basicamente da adesão aos bundles como medida preventiva da PAV.

$\mathrm{O}$ artigo de número $\mathrm{I}^{(19)}$, por exemplo, ao avaliar a adesão dos profissionais de saúde às boas práticas de prevenção da PAV, evidenciaram que o item com maior adesão foi a avaliação diária da sedação (91\%), seguido da troca do circuito a cada 7 dias $(82,6 \%)$. Em contrapartida, o item com menor adesão foi à manutenção da pressão do cuff entre 20 e $30 \mathrm{~cm} \mathrm{H} 20$ (23,9\%).

As medidas preventivas estão relacionadas com a queda nos índices de infecção, chegando a uma redução de densidade de incidência de 8,6 PAV por 1000 dias de ventilação para 2,0 PAV por 1000 dias de ventilação. Além disso, podem reduzir os dias de internação em UTI de 36 para 27 dias de permanência ${ }^{(6)}$.

Os autores ${ }^{(19)}$ avaliaram entre outros fatores de risco para a PAV o nível da profundidade da sedação conforme a escala de $\operatorname{RASS}^{(23)}$ em um estudo multicêntrico internacional, demonstrou que a proporção de pacientes com sedação profunda diminuiu de $55,2 \%$ para $44 \%$ após implantação de protocolo de sedação e analgesia gerenciado por enfermeiros.

Outra intervenção indicada no pacote de medidas para a prevenção da PAV em pacientes adultos, diz respeito à higiene oral com clorexidina $0,12 \%^{(14)}$ constataram em seus estudos que embora a higiene oral caracterize-se como um cuidado imprescindível em pacientes sob ventilação mecânica, os resultados obtidos demonstraram uma baixa adesão a esta medida $(48,8 \%)$, o que foi atribuído a falta de conhecimento dos profissionais, capacitação técnica inadequada e ausência de acompanhamento diário da adesão.

Os autores ${ }^{(24)}$ avaliaram a adesão ao conjunto completo de prevenção de PAV e identificaram conformidade de $94 \%$, repercutindo na redução da densidade de incidência da PAV de 18,5\% para 9\%, redução da mortalidade de $38 \%$ para $30 \%$, tempo de permanência na UTI de 2 dias e custos hospitalares de US\$2000 a US\$3000 por caso de PAV.

Os mesmos autores afirmaram que esse resultado só foi possível, graças à educação permanente e treinamentos periódicos, por meio de protocolo gerenciado rigorosamente, além do método observacional, considerado o mais preciso para o monitoramento da adesão ${ }^{(24)}$.

Diante do exposto, é relevante que ações de prevenção de PAV sejam promovidas nas instituições de saúde para a garantia da segurança dos pacientes. A participação da equipe multiprofissional também se faz significativa, através da adoção de medidas preventivas padronizadas, treinamento de equipes, educação continuada no processo assistencial, a fim de, evitar falhas neste processo e com isso, reduzir as taxas de mortalidade ${ }^{(25)}$.

Segundo ${ }^{(17)}$, em seu artigo, avaliou da mesma forma a adesão e conformidade das práticas que integram um bundle de prevenção da PAV. As práticas selecionadas 
para tal foram: 1 - posicionamento da cabeceira entre 30 e $45^{\circ} ; 2$ - pressão do cuff entre 20-30 cm H2O; 3 - higiene oral com clorexidina $0,12 \%$; 4 - fisioterapia respiratória e motora; 5 - cuidados com a aspiração das secreções; 6 - interrupção da infusão de sedativos.

A frequência de adesão ao bundle pelos profissionais teve significância para 3 dos 6 itens propostos, sendo eles: posição da cabeceira entre 30 a $45^{\circ}$, cuidados com aspiração das secreções e a fisioterapia respiratória e motora. A taxa de adesão geral foi de $77,4 \%{ }^{(17)}$.

Desde a criação do primeiro bundle, outros bundles de ventilação mecânica estão sendo propostos com sugestões de medidas de prevenção diferentes para efetivar a implementação. No entanto, é importante salientar, que o Institute for Healthcare Improvement (IHI) propõe que não sejam elaborados bundles muito extensos, devido à maior dificuldade em administrá-los e aplicálos tanto ao público alvo, como para os profissionais $^{(26)}$.

De igual modo, os autores ${ }^{(16)}$ avaliaram a adesão da enfermagem ao bundle antes e após educação permanente sobre o tema. As medidas preventivas realizadas pela enfermagem foram: posição do filtro do ventilador mecânico acima da inserção do tubo endotraqueal ou traqueostomia, ausente de sujidades; cabeceira do leito elevada entre $30^{\circ}$ e $45^{\circ}$; higiene oral com clorexidina $0,12 \%$ e escovação dos dentes registrados em prontuário nos horários de 8 e 20 horas; e pressão do balonete entre 20 e $30 \mathrm{mmHg}$. A adesão geral da equipe de enfermagem foi de $92,7 \%$, havendo aumento significativo na adesão após a Educação Permanente.
Já os autores ${ }^{(18)}$, no artigo de número IV, avaliou o impacto dos bundles e o aprendizado interprofissional, por meio de 3 etapas de pesquisa: pré-intervenção, que consistiu em observação direta e entrevista; intervenção, na qual o treinamento foi realizado por meio de simulações clínicas; e pós-intervenção, na qual foi avaliado o impacto das estratégias implementadas por observação direta.

Os autores do artigo IV se utilizaram de estratégia semelhante ao estudo ${ }^{(24)}$ para o monitoramento das ações preventivas. Além disso, após a intervenção, obtiveram aumento da adesão à pressão endotraqueal do cuff $(8,10 \%)$, interrupção diária da sedação $(16,67 \%)$, e aspiração subglótica $(18,75 \%)$.

Os autores $^{(7)}$, no artigo V, identificaram os procedimentos de enfermagem em doentes submetidos à ventilação mecânica invasiva a partir de normas publicadas em 2015 pela Direção Geral de Saúde de Portugal. Os cuidados de enfermagem que foram identificados e observados foram: elevação da cabeceira da cama, higienização das mãos, aspiração de secreções, higiene oral, manutenção dos circuitos ventilatórios. Sendo a verificação da pressão do cuff e a aspiração de secreções os procedimentos que registaram menor adesão entre os profissionais.

A partir da percepção dos profissionais de Enfermagem sobre a segurança do paciente sob ventilação mecânica com vistas à prevenção da PAV, o artigo $\mathrm{VI}^{(28)}$, evidenciou entre os entrevistados que recomendaram intervir nos fatores de risco modificáveis para a prevenção de PAV adotando medidas semelhantes as trazidas pelo estudo ${ }^{(13)}$ como: higienização das mãos; decúbito elevado entre 30 e $45^{\circ}$; adequação diária da sedação e do 
teste de respiração espontânea; aspiração subglótica; higiene oral com antissépticos; uso criterioso de bloqueadores neuromusculares; cuidados com o circuito do ventilador; inaladores e nebulizadores; cuidados para evitar a extubação não programada e a reintubação; monitorização da pressão de cuff; utilização preferencial de intubação orotraqueal; e manutenção da sondagem enteral na posição gástrica ou pilórica.

O estudo ${ }^{(20)}$ avaliou a conformidade de cinco medidas preventivas da PAV, a saber: elevação da cabeceira de $30^{\circ}$ a $45^{\circ}$, interrupção diária da sedação, profilaxia de úlcera péptica, profilaxia de trombose venosa profunda (TVP) e higiene oral com clorexidina. Destas, a medida mais realizada foi a profilaxia de úlcera péptica (conformidade superior a 80\%), e as realizadas com menor frequência foram a elevação da cabeceira e a higiene oral (conformidade inferior a 50\%).

$O$ estudo $^{(6)}$ destaca em seu artigo, número VIII desta revisão, que a estratégia de rounds, visita multiprofissional beira leito, foi descrita pela equipe multiprofissional como positiva troca de saberes, sendo um momento de discussão e proposição em conjunto de alternativas de cuidado, além da aplicação e verificação dos protocolos estabelecidos.

Os $\quad \operatorname{artigos}^{(21-22,29)}$, trazem contribuições importantes a respeito de evidências relacionadas a prevenção da PAV. O estudo ${ }^{(29)}$, por exemplo, ao avaliar o conhecimento dos profissionais acerca dessa temática evidenciou fragilidades dos participantes em relação a higiene das mãos e biossegurança. Para tal, recomendam estratégias para a melhor adesão à prática, fortalecendo os conceitos de periodicidade, momentos necessários e da própria técnica.

Nesse ínterim, a ANVISA recomenda fortemente a utilização de sabonete líquido com antissépticos como a clorexidina em locais com presença de bactérias multirresistentes, como as UTIs, com o objetivo de diminuir a transmissão cruzada ${ }^{(30)}$. Nas demais áreas de serviços de saúde, a utilização de preparação alcoólica para as mãos segue sendo estimulada.

O estudo ${ }^{(21)}$ traz considerações a respeito da realização da higiene oral relacionada à diminuição da incidência de PAV, mais precisamente sobre a escovação dentária que, apesar de diminuir o tempo de ventilação mecânica, pode levar ao deslocamento do biofilme formado na região oral para as vias aéreas inferiores.

Além disso, em relação à pressão do cuff, os mesmos autores ${ }^{(21)}$ consideram os valores entre 18 a $22 \mathrm{mmHg}$ ou 25 a $30 \mathrm{~cm}$ de H20 como mais adequados, a fim de evitar o comprometimento da microcirculação traqueal devido à pressão excessiva e à passagem de secreção subglótica na pressão insuficiente. Por outro lado, a ANVISA recomenda uma pressão entre 20 e $23 \mathrm{mmHg}$, por considerar valores acima destes, como potenciais riscos a lesões por isquemia e estenose de traqueia ${ }^{(30)}$.

O estudo ${ }^{(22)}$ contempla ainda a indicação do uso de filtro trocador de calor e umidade (Heat and Moisture Exchangers HME), e as indicações e os cuidados com o sistema de aspiração, inalação e nebulização como intervenções que causam impacto significativo na prevenção da PAV. 


\section{CONCLUSÃO}

Percebe-se que as medidas de prevenção e controle da PAV são bem disseminadas e apresentam um consenso no âmbito científico a partir das diretrizes nacionais e internacionais definidas. Os estudos analisados traziam em sua maioria o conjunto de medidas já amplamente divulgado e praticado por grande parte dos hospitais, em especial aqueles que apresentam setores de UTI.

O desafio está no acompanhamento da adesão dos profissionais a esse conjunto de ações, que por vezes é negligenciada pelo grande número de demandas assistenciais, reverberando diretamente nas taxas de infecção e em consequência na segurança do paciente e na qualidade do cuidado ofertado. Além disso, áreas que atuam diretamente na assistência ao paciente em ventilação mecânica, como Fisioterapia e Medicina, por exemplo, ainda publicam de forma tímida estudos sobre o tema, ficando a cargo da Enfermagem a maioria das evidências encontradas.

Assim, sugere-se que novos estudos sejam realizados na ótica interprofissional e que intervenções e instrumentos de análise da adesão das medidas de prevenção de PAV sejam difundidos e amplamente discutidos e replicados.

Considera-se como ponto limitante ao estudo a ausência de publicações de outras categorias profissionais além da Enfermagem, bem como a incipiente literatura a respeito do tema no ano de 2020, notadamente afetada pelo contexto de pandemia enfrentado por todos, mas que a priori deve trazer repercussões e inovações importantes a respeito do cuidado ao paciente em suporte ventilatório.

\section{REFERÊNCIAS}

1. Bastos ECB, Lima OS, Laurindo MV, Vasconcelos FF, Rocha RR. Perfil epidemiológico das infecções em uma unidade de terapia intensiva de emergência. Brazilian Journal of Health Review [Internet]. 2019 [acesso em 01 jan 2021]; 2(3):1654-60. Disponível em: https://www.brazilianjournals.com/index.php/ BJHR/article/view/1432/1549

2. Giroti ALB, Ferreira AM, Rigotti MA, Sousa AFL, Frota OP, Andrade D. Programas de Controle de Infecção Hospitalar: avaliação de indicadores de estrutura e processo. Revista da Escola de Enfermagem da USP [Internet]. 2018 [acesso em $30 \mathrm{dez}$ 2020]; 52:1-7. Disponível em: https://www.scielo.br/pdf/reeusp/v52/1980220X-reeusp-52-e03364.pdf

3. Alvim ALS. Qualidade dos programas de controle de infecção hospitalar: revisão integrativa. Revista Gaúcha de Enfermagem [Internet]. 2020 [acesso em $30 \mathrm{dez}$ 2020]; 41:1-10. Disponível em: https://www.scielo.br/pdf/rgenf/v41/pt_19831447-rgenf-41-e20190360.pdf

4. Brasil. Agencia Nacional de Vigilância Sanitária. Medidas de Prevenção de Infecção Relacionada à Assistência à Saúde, Anvisa, 2017. [acesso em $30 \mathrm{dez}$ 2020]. Disponível em:

https://www20.anvisa.gov.br/segurancadopaci ente/index.php/publicacoes/item/caderno-5.

5. Cavalcante ABL et al., Pneumonia associada à ventilação mecânica: consequências e mortalidade em uma unidade de terapia intensiva. Revista Eletrônica Acervo Saúde [Internet]. 2020 [acesso em 30 dez 2020]; (44):1-9. Disponível em: 
https://acervomais.com.br/index.php/saude/art icle/view/2385/1671

6. Liz JS, Gouvea PB, Acosta AS, Sandri JVA, Paula DM, Maia SC. Cuidados multiprofissionais relacionados a prevenção da pneumonia associada à ventilação mecânica. Enfermagem em Foco [Internet]. 2020 [acesso em $22 \mathrm{dez} 2020 ; 11(2): 85-90$. Disponível em: http://biblioteca.cofen.gov.br/wpcontent/uploads/2020/10/cuidadosmultiprofissionaisprevencao-pneumonia-associada-ventilacaomecanica.pdf

7. Martins PCAM, Vaz AKMG. Infecções prevalentes na unidade de terapia intensiva de um hospital universitário. Enfermagem Brasil [Internet]. 2020 [acesso em $30 \mathrm{dez}$ 2020]; 19(3):238-45. Disponível em: http://portalatlanticaeditora.com.br/index.php/ enfermagembrasil/article/view/3948/html\#: :t ext $=$

As $\% 20$ IRAS $\% 20$ s $\%$ C3\%A3o\%20considerad as\%20mais,de $\% 20$ procedimentos\%20invasiv os $\% 2$

0rotineiramente\%20realizados.

8. Bacellar KK, Furuya RK, Sanches JPS, Kerbauy G, Belei RA, Pieri FM. Associações demográficas e clínicas com pneumonia hospitalar adquirida e associada à ventilação mecânica. Advances in Nursing and Health [Internet]. 2020 [acesso em $30 \mathrm{dez}$ 2020]; 2:17-33. Disponível em: http://www.uel.br/revistas/uel/index.php/anh/ article/view/39649/28111

9. Barros MMA, Pereira ED, Cardoso FN, Silva RA. O enfermeiro na prevenção e controle de infecções relacionadas à assistência à saúde. Universitas: Ciências da Saúde [Internet]. 2016 [acesso em 30dez 2020]; 14(1):15-21. Disponível em: https://www.publicacoesacademicas.uniceub. br/cienciasaude/article/viewFile/3411/3066
10. Souza MT, Silva MD, Carvalho R. Revisão integrativa: o que é e como fazer. Einstein. [Internet]. 2010 [acesso em $17 \mathrm{dez}$ 2020]; 8(1):102-6. Disponível em: https://www.scielo.br/pdf/eins/v8n1/pt_16794508-eins-8-1-0102.pdf

11. Ursi ES. Prevenção de lesões de pele no perioperatório: revisão integrativa da literatura. [dissertação]. Ribeirão Preto: Universidade de São Paulo, Escola de Enfermagem de Ribeirão Preto; 2005.

12. Gallacher JA. Implementation of ventilator-associated pneumonia clinical guideline (Bundle). The Journal for Nurse Practitioners [Internet]. 2012 [acesso em 30 dez 2020]; 8(5):377-82. Disponível em: https://www.sciencedirect.com/science/article /abs/pii/S1555415512001080

13. Dudeck MA et al., National Healthcare Safety Network (NHSN) report, data summary for 2012, Device-associated module. American Journal of Infection Control [Internet]. 2012 [acesso em 01 jan 2021]; 41(12):1148-66. Disponível em: https://pubmed.ncbi.nlm.nih.gov/24274911/

14. Chicayban LM, Terra ELVS, Ribela JS, Barbosa PF. Bundles of pneumonia associated with mechanical ventilation: the importance of multidisciplinarity. Perspectivas Online: Biológicas e Saúde [Internet]. 2017 [acesso em 01 jan 2021]; 7(25):25-35. Disponível em: http:/www.

seer.perspectivasonline.com.br/index.php/biol ogicas_e_saude/article/view/1200

15. Leal GA, Ribeiro JB, Dos Santos JJ, Cavalcante AB. Cuidados de enfermagem para prevenção da pneumonia associada à ventilação mecânica em unidades de terapia intensiva: uma revisão literária. Cadernos de Graduação - Ciências Biológicas e da Saúde (UNIT) [Internet]. 2017 [acesso em 01 jan 2021]; 4(1):95-108. Disponível em: 
https://periodicos.set.edu.br/cadernobiologica s/article/view/3657/2166

16. Branco A et al., Education to prevent Ventilator associated pneumonia in Intensive Care Unit. Revista Brasileira de Enfermagem [Internet]. 2020 [acesso em $22 \mathrm{dez}$ 2020]; 73(6)e20190477. Disponível em: http://dx.doi.org/10.1590/0034-7167-20190477

17. Barros FRB. Adesão ao bundle de prevenção de pneumonia associada à ventilação mecânica. Revista Cuidarte [Internet]. 2019 [acesso em $22 \mathrm{dez}$ 2020]; 10(2):1-9. Disponível em: http://dx.doi.org/10.15649/cuidarte.v10i2746

18. Coelho AFP, E Vieira RAM, Leite MA, Lucas TC. O impacto da aprendizagem interprofissional na pneumonia associada à ventilação: implementação de bundles em uma unidade de cuidados intensivos. Enfermagem em Foco [Internet]. 2019 [acesso em 22 dez 2002]; 10(4):93-100. Disponível em:

http://revista.cofen.gov.br/index.php/enferma gem/article/view/2262/611

19. Alecrim RX et al., Boas práticas na prevenção de pneumonia associada à ventilação mecânica. Acta paulista de Enfermagem [Internet]. 2019 [acesso em 22 dez 2020]; 32(1):11-7. Disponível em: http://www.scielo.br/scielo.php?script=sci_art text\&amp;pid=S0103-

21002019000100011\&amp;lng=en\&amp;nrm =isso

20. Frota ML et al., Good practices for preventing ventilator-associated pneumonia in the emergency department. Revista da Escola de Enfermagem da USP [Internet]. 2019 [acesso em 22 dez 2020]; (53)e0460. Disponível em:
21. Silva Lourençone, EM, Branco A, Monteiro AB, Fonseca JP, Caregnato RCA. Adesão às medidas preventivas versus incidência de pneumonia associada à ventilação mecânica. Revista de Epidemiologia e Controle de Infecção. [Internet]. 2019 [acesso em $22 \mathrm{dez}$ 2020]; 9(2). Disponível em: https://online.unisc.br/seer/index.php/epidemi ologia/article/ view/12596

22. Zigart JAA et al., Adesão ao protocolo de pneumonia associado à ventilação mecânica. Revista de Enfermagem UFPE online [Internet]. 2019 [acesso em $22 \mathrm{dez}$ 2020]; 13(3):655-63. Disponível em: https://periodicos.ufpe.br/revistas/revistaenfer magem/article/view/234873/31551

23. Sachetti A et al., Adherence to the items in a bundle for the prevention of ventilatorassociated pneumonia. Revista Brasileira de Terapia Intensiva [Internet]. 2014 [acesso em 22 dez 2020]; 26(4):355-9. Disponível em: https://www.scielo.br/pdf/rbti/v26n4/0103507X-rbti-26-04-0355.pdf

24. Samra SR, Sherif DM, Elokda AS. Impact of VAP bundle adherence among ventilated critically ill patients and its effectiveness in adult ICU. The Egyptian Journal of Chest Diseases and Tuberculosis [Internet] 2017 [acesso em 1 jan 2021]; 66(1):81-6. Disponível em: https://www.sciencedirect.com/science/article /pii/S042276381630200X\#: :text=Male\%20a nd $\%$

20medical\%20patients\%20were,days $\% 20$ subs equently $\% 20$ lowering $\% 20$ the $\% 20$ cost.

25. Pereira GTRN et al., Estratégias e conhecimento profissional sobre as medidas de prevenção da pneumonia associada à ventilação mecânica: uma revisão integrativa. Rev Interdisc Ciências e Saúde [Internet]. 2017 [acesso em 02 jan 2021]; 4(2):121-33. Disponível 
https://revistas.ufpi.br/index.php/rics/article/vi ew/6023/3921

26. Ficagna FT, Valcarenghi RV, Demarche NF, Ferreira D. O Impacto da utilização de bundles na prevenção da pneumonia associada à ventilação mecânica: uma revisão integrativa. Rev Enferm. Atual In Derme [Internet]. 2020 [acesso em 02 jan 2021]; 92(30): 6-20. Disponível em: revistaenfermagematual.com.br/index.php/rev ista/article/view/468/650

27. Cruz JRM, Martins MDS. Pneumonia associada à ventilação mecânica invasiva: cuidados de enfermagem. Revista de Enfermagem Referência [Internet]. 2019 [acesso em 22 dez 2020]; 4(20):87-96. Disponível em: http://www.scielo.mec.pt/scielo.php?script=sc i_arttext\&amp;pid=S0874-

02832019000100010\&amp;lng=pt\&amp;nrm =iso

28. Dutra LA et al., Pneumonia associada à ventilação mecânica: percepção dos profissionais de enfermagem. Revista de Enfermagem UFPE online [Internet]. 2019 [acesso em 22 dez 2020]; 13(4):884-92. Disponível em: https://periodicos.ufpe.br/revistas/revistaenfer magem/article/view/237363/31695

29. Melo MM, Santiago LMM, Nogueira DL, Vasconcelos MFP. Ventilator-Associated Pneumonia: the Knowledge of Health Professionals Towards Prevention and Educational Measures.Revista de Pesquisa: Cuidado é Fundamental (online) [Internet]. 2019 [acesso em $22 \mathrm{dez}$ 2020]; 11(esp):37782

http://dx.doi.org/10.9789/2175-

5361.2019.v11i2.377-382

30. ANVISA. Agência Nacional de Vigilância Sanitária. Medidas de Prevenção de Infecção Relacionada à Assistência à Saúde. Brasília DF. 1a ed. 2013. Disponível em: https://www.segurancadopaciente.com.br/wpcontent/uploads/2015/09/ebook-anvisa-04medidas-de-prevencao-de-de-infeccaorelacionada-a-assistencia-a-saude.pdf

Submissão: 2021-02-09

Aprovado: 2021-04-16 